American Journal of Applied Sciences 9 (1): 141-151, 2012

ISSN 1546-9239

(C) 2012 Science Publications

\title{
Performance Evaluation of Wireless Sensor Network Channel in Agricultural Application
}

\author{
${ }^{1}$ Naseer Sabri, ${ }^{1}$ S.A. Aljunid, ${ }^{1}$ R.B. Ahmad, \\ ${ }^{1}$ M.F. Malek, ${ }^{1}$ Abid Yahya, ${ }^{2}$ R. Kamaruddin and ${ }^{3}$ M.S. Salim \\ ${ }^{1}$ Department of Communication Engineering, \\ ${ }^{2}$ Department of Bioprocess Engineering, \\ ${ }^{3}$ Department of Mechatronics Engineering, \\ Computer and Communication Engineering School, \\ University Malaysia Perlis, Malaysia
}

\begin{abstract}
Problem statement: In this study, the behavior of small scale fading channel due to a moving sink node of a Wireless Sensor Network (WSN) or moving of surrounding objects is presented. Approach: Specifically, this research introduces the effects of multipath and scattering on the transmitted signal due to mobile sink node within forest or closed field filled of reflectors and scatters. Signal propagation above the cross canopy suffer attenuation and variance in the strength of the received signal. Results: The density of leaves in the crop changes over time thus results in more signal obstacles and attenuation at receiver node. The sink node is considered to be in stationary status or it moves with speed not more than $1 \mathrm{~m} \mathrm{sec}^{-1}$, while the sensor nodes of the WSN is preplanned in a fixed position. Conclusion/Recommendations: The modeling of pass band transmitted signal fused in Rayleigh fading channel of stationary and moving status of a receiver are derived. The fluctuation of RF signal, average received signal level, outage probability and the Doppler shift effects are simulated and a detailed analysis is present. The appropriate spatial distribution of the network nodes and the exist of a LOS component will highly necessary between sensor nodes and the sink node in agricultural applications. Indeed power consumption must well maintain so packet loss due to less power level can be overcome. Best position found is center of the application field of the greenhouse for the sink node in stationary situation, while for movement situation the best height of the sink node is to be little above line level of the sensor nodes.
\end{abstract}

Key words: Wireless Sensor Network (WSN), fading channel, doppler shift, Line of Sight (LOS), outage probability

\section{INTRODUCTION}

Wireless sensor networks are part of a growing collection of information technology, which are moving away from the traditional desktop wired network architecture towards more ubiquitous and universal mode of information connectivity. It consists of spatially distributed autonomous sensor nodes to monitor physical or environmental conditions, such as temperature, humidity, vibration, sound, pressure, gases, motion or pollutants and to cooperatively flow their data through the network routes to a base station. The new modern networks are bi-directional, enabling also to control the activity of the nodes. The WSN consist of nodes, from a few to several hundreds or thousands, where each node is connected to one or several sensors. Sensor node essentially combines several parts: a transceiver unit with a proper antenna type either internal or external, a microcontroller unit, an electronic circuitry to interface various sensors and an energy source, such as battery or an embedded form of energy harvesting. The size of a sensor node might vary in size from that of large down to the size of a grain of dust, although functioning "motes" of genuine microscopic dimensions have yet to be created. Size and cost constraints on sensor nodes result in corresponding constraints on resources such as energy, memory, computational speed and communications bandwidth. WSNs topology can vary from a simple star network to an advance mesh multi hop wireless network, where the data propagation technique through network nodes can be routing or flooding

Corresponding Author: Naseer Sabri, Department of Communication Engineering, Computer and Communication Engineering School, University Malaysia Tel: +60192529327 Fax: +60163105440 
(Dargie and Poellabauer, 2010; Sohraby et al., 2007). Figure 1 depicts the simple star and multi hop sensor-actor network structure. The main characteristics of a WSN are:

- Power consumption limits for nodes depending on batteries or energy harvesting

- Reliability of the network for node failures events

- Mobility of base station node or network nodes

- Various types of sensing or actor nodes

- Scalability to large scale of nodes deployment

- Ability to work in harsh environmental conditions

- Flexibility of use and installation

- Autonomous operation

The base stations are one or more main components of the WSN with much more computational, energy and communication resources. They typically forward data from the WSN on to a server; thus they act as a gateway between sensor nodes of the network and the remote user. Also they can embed with computational/control algorithms to acts as a network brain that can do monitoring and acting tasks (Sabri et al., 2011).

Wireless channels have adverse properties, such as path loss, multi-path fading, adjacent channel interference, Doppler shifts and half-duplex operations (Willig et al., 2005). The low power communication and the node mobility affect the WSNs and deviate it to be notoriously unpredictable and inherently unreliable. Therefore, the Quality of Service (QoS) of the network cannot be always guaranteed. As a result, the applications will suffer from packet loss and time-varying delay, both of which could significantly degrade the system performance, or even cause system instability. The channel modeling of WSNs featuring node mobility is investigated in this study. The overall goal is to explore the effects of motion of the nodes on the received signal. Hence a proper selection of a receiver with high sensitivity to ensure better reliability and performance of the network so that the required performance of applications is guaranteed in dynamic, noisy environments. In particular, the effects of multipath and Doppler shift on communication signals in WSN with mobile sink node and dense of foliage is presented. Depending on the application, nodes in a WSN can be either stationary or mobile.

In many situations, however, sensor nodes are stationary whereas base station node, e.g., mobile robots and unmanned aerial vehicles, are mobile. Indeed movement of surrounding object could result in same effects of mobility of nodes.

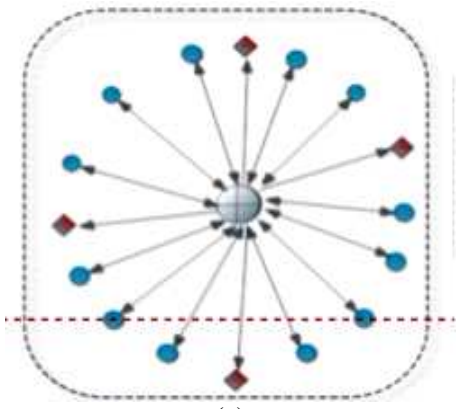

(a)

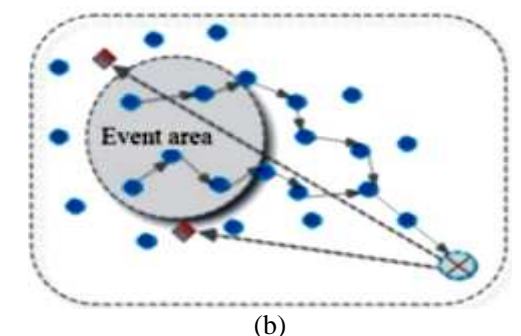

Fig. 1: (a) Sink based star WSN topology, (b) Multi hop sink based WSN topology

Table 1: Comparison between Bluetooth and ZigBee

\begin{tabular}{lll}
\hline & Bluetooth & ZigBee \\
\hline Standards & IEEE 802.15 .1 & IEEE 802.15 .4 \\
Frequencies & $2.4 \mathrm{GHz}$ & $2.4 \mathrm{GHz}$ \\
Data rate & $1 \mathrm{Mb} \mathrm{s}-1$ & $20-250 \mathrm{~kb} \mathrm{~s}-1$ \\
Latency (time to establish a new link) & $<10 \mathrm{~s}$ & $30 \mathrm{~m} \mathrm{sec}$ \\
Modulation technique & FHSS 2 & DSSS 1 \\
No. of nodes & 8 & 65,000 \\
Network topology & Ad hoc piconets & Ad hoc, star, mesh \\
Range & $8 \mathrm{~m}($ Class II, III) & to \\
$100 \mathrm{~m}$ (Class I) & $1-100 \mathrm{~m}$ & \\
Data type & Audio, graphics, \\
pictures, files & Small data packet \\
Battery life & 1 week & $>1$ year \\
Extendibility & No & Yes \\
\hline${ }^{1}$ DSSS: Direct Sequence & Spread Spectrum. ${ }^{2}$ FHSS: Frequency \\
Hopping Spread Spectrum & \multicolumn{2}{l}{}
\end{tabular}

Sensor nodes are usually low-cost, low-power; small devices equipped with limited sensing, data processing and wireless communication capabilities, while actuator nodes typically have stronger computation and communication powers and more energy Budget that allows longer battery life (Melodia et al., 2007; Balamurugan and Duraiswamy, 2011). Regardless, resource constraints apply to both sensors and actuators.

WSN technology: There are two standard technologies are available for WSN: ZigBee and Bluetooth. They are operating at $2.4 \mathrm{GHz}$ of the Industrial Scientific and Medical (ISM) license free band, huge spectrum allocation and worldwide compatibility (Kirubanand and Palaniammal, 2011; Patel et al., 2006). Table 1 shows the main features of Bluetooth and Zigbee 
Am. J. Applied Sci., 9 (1): 141-151, 2012

technologies. In wireless communication, increasing the frequency causes bandwidth to be increased, thus allowing for higher data rates with high power requirements over transmission distance is considerably shorter (Callaway 2004; Shan et al., 2004).

The traditional single hop communication consumes higher power than Multi-hop communication over the ISM band; therefore the multi hop communication might well possible for WSN (Ismail and Othman, 2009; Shan et al., 2004). WSN can be created using IEEE 802.11$\mathrm{WiFi}$, but this protocol is developed in PC based systems and its power consumption is rather high and the short autonomy of a battery power supply still remains an important disadvantage (ANSI/IEEE Std 802.11, 1999; Anastasi et al., 2009).

Physical aspects of applying WSN in agriculture: Real time radio propagation in real environment is complex due to the existing of multipath propagation, shadowing and attenuation. In agriculture field, the wireless communication faces challenges in terms of nodes location for wide area mesh coverage and reliable communication link quality above crop canopies. WSN must has the ability to work in a wide range of environments such as bare fields, orchards, vineyards from flat to complex topography and with various weather conditions, all of which affect radio performance (Andrade-Sanchez et al., 2007). In these environment and situations, the terrain, crop growth, nodes spacing, antenna height and in addition to more common factors will affects the link power budget (Tate et al., 2008). For applications inside buildings like greenhouses or warehouses, the radio signal has to go many objects like windows, walls, pallets, machines, which indeed cause a significant degradation in received signal strength. Mostly a 10-20 dB of a received signal level above the sensitive threshold of the receiver is an accepted value for the link budget (Tate et al., 2008). As the crop growth, the density of the leaves increased over time and thus message rate decreases, while when there are less leaves the message rate increases. Signal propagation above the cross canopy results in attenuation and variance in the received signal strength (Hebel, 2006). Hebel shows that attenuation and signal strength variance were dependent on line of sight losses and heights less than the Fresnel zone radius (Hebel et al., 2007). Experimentation in mature corn fields ( $2.5 \mathrm{~m}$ high) with transceivers placed at antenna heights of 1.5 and $2 \mathrm{~m}$. and a distance of $100 \mathrm{~m}$, showed an average $10 \mathrm{~dB}$ loss when the transceivers were placed in or across the corn rows (Tate et al., 2008).

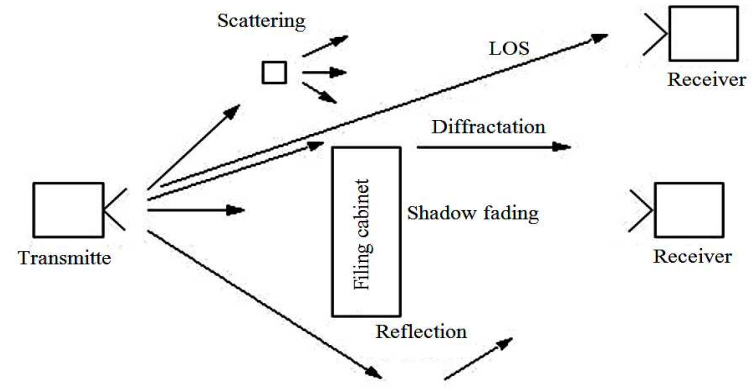

Fig. 2: Multipath propagation

Propagation problems: Nowadays the huge conversion of using wireless sensor networks which are almost embedded within all application fields needs a thorough investigation on channel performance prior to the implementation. In mobile communication, a principle problem with the propagation environment is the rapid variation in received signal strength as the communicating parties move as well as the surrounding objects.

RF signal propagates from the transmitter to the receiver via multiple different paths due to the obstacles and reflectors existing in the wireless channel. These multipath are caused by mechanisms of reflection, diffraction and scattering from buildings, structures, trees, foliage and other obstacles in the propagation environment (Chomphan, 2010; Andersen et al., 1995; Tse and Viswanath, 2005). Figure 2 shows the multipath propagation based on Line of Sight (LOS) path and Non Line of Sight (NLOS) paths.

A typical instant of fading channel is the mobile radio channel, where the mobile receiver antenna collects up multipath and scattering reflections. Thus, a time varying behavior in the received signal power will characterize the mobile channel. When the network nodes are far away, the Line of Sight (LOS) signal path does not exist and reception occurs mainly from the indirect signal paths. These multiple paths have different propagation lengths and thus will cause amplitude and phase fluctuations and time delay in the received signal. Therefore, the main effect of multipath propagation can be described in terms of fading and delay spread (Haykin and Moher, 2009).

Agricultural and environmental monitoring represents a class of wireless sensor network applications with enormous potential benefits for scientific community. Instrument the environment with numerous networked miniature sensors can enable long term data collection at scales and resolutions that are difficult, if not impossible, to obtain otherwise.

The ability to communicate not only allows sensor data and control information to be communicated 
across the network of nodes, but nodes to cooperate in performing more complex tasks, such as statistical sampling, data aggregation and system health and status monitoring (Sabri et al., 2011; Mainwaring et al., 2002).

This study targets to investigate the small scale fading effects of WSN on modulated pass band signal by employing MFSK modulation technique, it consider the mobile sink node unit as stationary and as moving status with consideration that surrounding objects may be locally spatial change and of dense foliage and plants.

Wireless channel modeling: Multilevel Frequency Shift Keying (MFSK) Modulation Technique occurs when $f(t)$ takes on equiprobable values that $\left(3 \times 10_{8} \mathrm{~m}\right.$ $\sec ^{-1}$ ), in each symbol interval $T_{s}$ where the frequency spacing $\Delta \mathrm{f}$ is related to the frequency modulation index $h=\Delta f$. As such, $f(t)$ is modeled as a random pulse stream, that is Eq. 1:

$$
f(t)=\sum_{n=-\infty}^{\infty} f_{n} p\left(t-n T_{s}\right)
$$

where, $f(t)$ is the information frequency in the $n^{\text {th }}$ symbol interval $n T_{s} \leq t \leq(n+1) T_{s}$ ranging over the set of $\mathrm{M}$ possible values $\xi_{\mathrm{i}}$ as above and $\mathrm{p}(\mathrm{t})$ is a unit amplitude rectangular pulse of duration $\mathrm{T}_{\mathrm{s}}$ seconds. Thus the complex signal transmitted in the $\mathrm{n}^{\text {th }}$ symbol interval is Eq. 2:

$$
\mathrm{s}^{\sim}(\mathrm{t})=\mathrm{A}_{\mathrm{c}} \ell^{\mathrm{j}\left[2 \pi\left(\mathrm{f}_{\mathrm{c}} \mathrm{t}+\mathrm{f}_{\mathrm{n}}\left(\mathrm{t}-\mathrm{n} \mathrm{T}_{\mathrm{s}}\right)\right)+\phi_{\mathrm{c}}\right]}
$$

The complex baseband modulation $\mathrm{s}^{\sim}(\mathrm{t})=\mathrm{A}_{\mathrm{c}} \ell^{\mathrm{j} 2 \pi \mathrm{f}_{\mathrm{n}}\left(\mathrm{t}-\mathrm{T}_{\mathrm{s}}\right)}$ is not constant over the same interval but rather has a sinusoidal variation. After demodulating with the complex conjugate of $\tilde{c_{r}}(t)$ at the receiver, we obtain Eq. 3:

$$
\mathrm{x}^{\sim}(\mathrm{t})=\mathrm{A}_{\mathrm{c}} \mathrm{e}^{\mathrm{j} 2 \pi \mathrm{f}_{\mathrm{n}}\left(\mathrm{t}-\mathrm{T}_{\mathrm{s}}\right)}+\mathrm{N}^{\sim}(\mathrm{t})
$$

For orthogonal signaling where the crosscorrelation Eq. 4:

$$
x^{\sim}(t)=A_{c} e^{j 2 \pi f_{n}\left(t-T_{s}\right)}+N^{\sim}(t)
$$

The frequency spacing is chosen such that $\Delta \mathrm{f}$ $=\mathrm{N} / \mathrm{T}_{\mathrm{S}}$ with integer. The bandwidth BW of MFSK is Eq. 5:

$$
\mathrm{BW}=2^{\mathrm{N}+1} \mathrm{f}_{\mathrm{b}} / \mathrm{N}
$$

Where:

$\mathrm{N}=$ The number of bits per symbol

$f_{b}=$ The bit rate of the data (Bhattacharya, 2005)

The baseband signal is modulated as a MFSK signal and sent over the channel. Equation 1-8 shows the orthognal baseband modulated signal mathmatical modeling and the bandwidth. The sensor nodes use the data of the environement sensing and coded it as MFSK signal to be flow through the channel over to the sink node of the WSN.

Channel fading: In mobile environment, fading can be categorized into large-scale fading and small-scale fading. Large-scale fading represents the average signal power attenuation or path loss due to motion over large areas, whereas small scale fading represents the shortterm fluctuation in the signal amplitude due to multipath. Small scale fading can be further classified as flat or frequency selective fading (Sklar, 1997). When there is a constant gain and a linear phase response over a bandwidth larger than the bandwidth of the transmitted signal, a received signal is said to undergo flat fading. Under these conditions, the received signal has amplitude fluctuations due to the variations in the channel gain over time due to multipath effects (Tse and Viswanath, 2005; Sklar, 1997). While if the mobile radio channel has a constant gain and linear phase response over a bandwidth smaller than that of the transmitted signal, the transmitted signal is said to undergo frequency selective fading (Sklar, 1997; Dersch and Ruegg, 1993). Reduction of the signal strength at the mobile unit occurs and causes significant fluctuations in the received signal amplitude with time leading to a phenomenon known as multipath fading or small scale fading. Figure 3 shows multipath effects on the power of a received signal.

Small-scale fading is also called Rayleigh fading because if the multiple reflective paths are large in number and there is no line-of-sight signal component, the envelope of the received signal is statistically described by Rayleigh distribution. When there is a dominant non fading signal component present, such as a line-of-sight propagation path, the small scale fading envelope is described by Rician distribution and, thus, is referred to as Rician fading (Tse and Viswanath, 2005; Papoulis, 1991). 


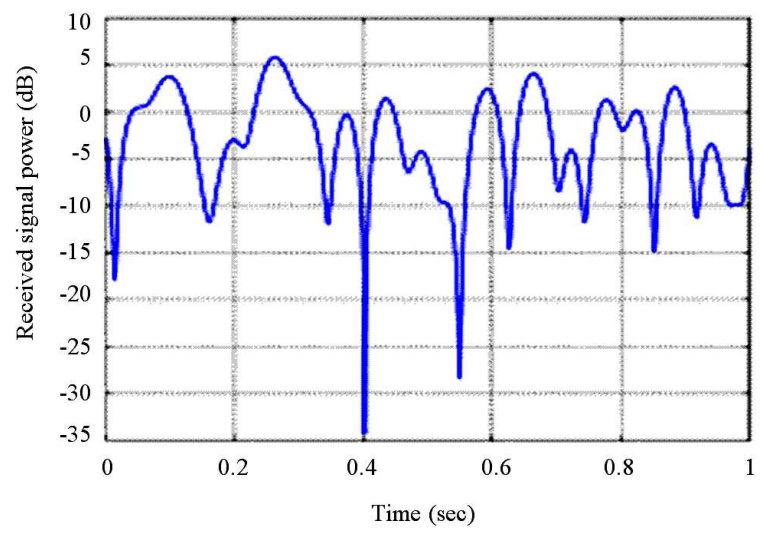

Fig. 3: Multipath fading signal

Signals traveling along different paths can have different Doppler shifts, corresponding to different rates of change in phase. The difference in Doppler shifts between different signal components contributing to a single fading signal component is known as the Doppler spread. Channels with a large Doppler spread have signal components that are each changing independently in phase over time (Haykin and Moher, 2009; Sklar, 1997). If the Doppler spread is significant relative to the bandwidth of the transmitted signal, the received signal will undergo fast fading. On the other hand, if the Doppler spread of the channel is much less than the bandwidth of the baseband signal, the signal undergoes slow fading (Sklar, 1997). Because of multiple reflections of the transmitted signal may arrive at the receiver at different times, this can result in InterSymbol Interference (ISI) due to the crashing of bits into one another. This time dispersion of the channel is called multipath delay spread and is an important parameter to evaluate the performance capabilities of wireless communication systems (Papoulis, 1991; Beaulieu and Cheng, 2001).

The model assumes a fixed transmitter, represented by the spatial fixed sensor nodes of the network, with vertical polarized antenna. The filed incident on the mobile antenna is assumed to be comprised of $\mathrm{N}$ plane waves with arbitrary carrier phases, arbitrary azimuthally angles of arrival with amplitude varying randomly (Sklar, 1997; Dersch and Ruegg, 1993). The movement is modeled as the movement of surrounding objects with fixed preplanned locations of network nodes or the movement of the sink node while it collects data from the sensor nodes. . Movement of dense plants and foliage surrounding network nodes is less to effects the received signals, thus only the movement of the sink node will be consider in this study.
Rayleigh stationary receiver fading model: The modulation of M-ary FSK will be used to encode the raw data into $\mathrm{M}$ different tone according to symbol data. Where $f_{c}$ is the carrier frequency and $f_{m}$ is the modulated frequency of $\mathrm{m}^{\text {th }}$ symbol, $\mathrm{m}=1,2, . . \mathrm{M}$. The transmitted band pass signal as in (2).

The mobile antenna, instead of receiving the signal over one line of sight, it will receive $\mathrm{N}$ of reflected and scattered waves of the transmitted signal. The phase and the instantaneous received power become random variables due to the different path lengths taken by the waves (Dersch and Ruegg, 1993; Beaulieu and Cheng, 2001). Assume no line of sight component, the received signal $\mathrm{s}(\mathrm{t})$ can be:

$\mathrm{s}_{(\mathrm{t})}=\operatorname{Re}\left\{\sum_{\mathrm{i}=1}^{\mathrm{N}} \mathrm{a}_{\mathrm{i}} \sigma^{\mathrm{j} 2 \pi\left(\mathrm{f}_{\mathrm{c}}+\mathrm{f}_{\mathrm{m}}\right) \mathrm{t}+\phi_{\mathrm{i}}}\right\}, \mathrm{nT}_{\mathrm{s}} \leq \mathrm{t} \leq(\mathrm{n}+1) \mathrm{T}_{\mathrm{s}}$

where, the number of paths is denoted by N. $\mathrm{f}_{\mathrm{m}}$ is the MFSK tone corresponding to symbol $\mathrm{m}$. The change in path length is encoded by the phase $\phi_{\mathrm{i}}$, when the path length changes by a wavelength, the phase change by $2 \pi$. Therefore the phases are uniformly distributed over $[0,2 \pi]$.

Rayleigh moving receiver fading model: In real world communication scenarios, the transmitter or the surrounding objects may be in motion. In static situation, the sink node and sensor nodes are fixed in position and the direct line of sight between the transmitter and receiver may be obstructed by plants and dense of foliage. At ultra high frequencies and above, therefore, the mode of propagation of the electromagnetic energy from transmitter to receiver is largely affected by the way of scattering (Sklar, 1997; Zheng and Xiao, 2003). The amplitude fluctuations of the received signal have been shown to follow Rayleigh distribution in the communication scenarios below. If the network node antenna receives a large number of reflected and scattered waves, the Instantaneous received power becomes a random variable, depending on the location of the antenna.

Let the $\mathrm{n}^{\text {th }}$ wave with amplitude $\mathrm{c}_{\mathrm{n}}$ and phase $\phi_{\mathrm{n}}$ arrive from an angle $\mathrm{s}(\mathrm{t})$ relative to the direction of the motion of the antenna. The Doppler shift of this wave is:

$\mathrm{Ds}_{\mathrm{n}}=\frac{\mathrm{v}}{\lambda} \cos \alpha_{\mathrm{n}}=\frac{\mathrm{w}_{\mathrm{c}} \mathrm{v}}{\mathrm{c}} \cos \alpha_{\mathrm{n}}$

Where:

$\mathrm{v}=$ The speed of the antenna 
$\mathrm{c}=$ The speed of light $\left(3 \times 10^{8} \mathrm{~m} \mathrm{sec}^{-1}\right)$

$a_{n}=$ Uniformly distributed over $[0,2 \pi]$

The received signal $0<$ speed $<1 \mathrm{~m} \sin ^{-1}$ (1) can be modified to include Doppler shift affects and expressed as Eq. 8-11:

$$
s(t)=\operatorname{Re}\left\{\sum_{n=1}^{N} a_{n} \sigma^{j 2 \pi\left(f_{c}+f_{m}+D s_{n}\right) t}\right\}
$$

We can simplify $s(t)$ let $W_{m n}=2 \pi\left(f_{m}+D s_{n}\right) t$ :

$$
\begin{aligned}
& s(t)=\operatorname{Re}\left\{\sum_{n=1}^{N} a_{n} \ell^{j\left(W_{c}+W_{m n}\right) t+\phi_{n}}\right\} \\
& s(t)=\operatorname{Re}\left\{\left[\sum_{n=1}^{N} a_{n} \ell^{j W_{m n} t+\phi_{n}}\right] \ell^{j W_{c} t}\right\} \\
& s(t)=s_{I} \cos 2 \pi f_{c} t-s_{Q} \sin 2 \pi f_{c} t
\end{aligned}
$$

where, the in-phase and quadrature components are given by Eq. 12 and 13:

$$
\begin{aligned}
& s_{I}(t)=\sum_{n=1}^{N} a_{n} \sigma^{j 2 \pi f_{m n} t+\phi_{n}} \\
& s_{Q}(t)=\sum_{n=1}^{N} a_{n} \sigma^{j 2 \pi f_{m n} t+\phi_{n}+\pi / 2}
\end{aligned}
$$

From (11-13), it is seen that the received signal is like a quadrature modulation carrier, the envelope of the received signal is given by Eq. 14:

$$
\operatorname{env}(t)=\left[s_{I}^{2}(t)+s_{Q}^{2}(t)\right]
$$

If $\mathrm{N}$ is large then we can invoke the central limit theorem and the fact that amplitude and the phase are independent for different components in order to approximate $\mathrm{s}_{\mathrm{I}}(\mathrm{t})$ and $\mathrm{s}_{\mathrm{Q}}(\mathrm{t})$ as jointly Gaussian random processes. Hence, It can be shown that the probability density function (pdf) of the envelope env (t) of the received signal is following the Rayleigh distributed (Beaulieu and Cheng, 2001; Zheng and Xiao, 2003) Eq. 15:

$$
f(\text { env })=\frac{\text { env }}{\sigma^{2}} \ell^{\frac{e n v^{2}}{2 \sigma^{2}}}
$$

Where:

$\sigma=$ The rms value of the received voltage is signal before envelope detection

$\sigma^{2}=$ The time average power of the received signal before envelope detection

The instantaneous power of the received signal is given by Eq. 16:

$\mathrm{P}(\mathrm{t})=(\mathrm{env})^{0.5}$

And average power received is Eq. 17:

$\mathrm{P}_{\mathrm{av}}=\operatorname{mean}\langle\mathrm{p}(\mathrm{t})\rangle$

Rician fading model: The Rician distribution is observed when, in addition to the multipath components, there exist a direct path. In the presence of such a path, the transmitted signal given in (8) can be written as Eq. 18:

$s(t)=\sum_{t=1}^{N} a_{t} \cos \left(w_{c} t+w_{m t} t+w_{d t} t+\varnothing_{t}\right)$

$+k_{d} \cos \left(w_{c} t+w_{d} t\right)$

where, the constant $\mathrm{k}_{\mathrm{d}}$ is the strength of the direct component, $\mathrm{w}_{\mathrm{d}}$ is the Doppler shift along the LOS path and $\mathrm{w}_{\mathrm{dt}} \mathrm{is}$ the Doppler shifts along the indirect paths. The envelope in this case has a Rician density function given by Eq. 19:

$f(r)=\frac{r}{\sigma^{2}} \exp \left(-\frac{r^{2}+k_{d}^{2}}{2 \sigma^{2}}\right) I_{o}\left(\frac{r k_{d}}{\sigma^{2}}\right), r \geq 0$

where, $I\left(\right.$,)is the zero ${ }^{\text {th }}$ order modified Bessel function of the first kind. The cumulative distribution of the Rician random variable is given as Eq. 20:

$\mathrm{F}(\mathrm{r})=1-\mathrm{Q}\left(\frac{\mathrm{k}_{\mathrm{d}}}{\sigma^{2}}, \frac{\mathrm{r}}{\sigma}\right), \mathrm{r} \geq 0$

where, Q(,)is the Marcum's Q function. The Rician distribution is often described in terms of the Rician factor $\mathrm{K}$, defined as the ratio between the deterministic signal power (from the direct path) and the diffuse signal power (from the indirect paths). $\mathrm{K}$ is usually expressed in decibels as Eq. 21:

$K(d B)=10 \log _{10}\left(\frac{k_{d}^{2}}{\sigma^{2}}\right)$ 
In Eq. 9, if $\mathrm{k}_{\mathrm{d}}$ goes to zero or if $\left(\mathrm{k}_{\mathrm{d}}^{2} / \sigma^{2}<<\mathrm{r}^{2} / 2 \sigma^{2}\right)$ the direct path is eliminated and the envelope distribution becomes Rayleigh, with $\mathrm{k}(\mathrm{dB})=-\infty$.

Outage probability: One of the most important consequences of Doppler fading is that the signal will experience deep fades occasionally as the mobile unit is in motion (Zheng and Xiao, 2003). The transmitted signal suffers deep fades that can lead to a complete loss of the signal or outage of the signal. The outage probability is a measure of the quality of the transmission in a mobile radio system. Outage occurs when the received signal power drops below a certain threshold level (Dersch and Ruegg, 1993; Zheng and Xiao, 2003). The rate at which the envelope crosses a specified signal level $\mathrm{P}_{\text {th }}$ in the positive direction can be calculated as Eq. 22:

$$
\mathrm{P}_{\text {out }}=\int_{0}^{\mathrm{p}_{\text {th }}} \mathrm{p}(\mathrm{t}) \mathrm{dt}
$$

where, $\mathrm{P}_{\text {th }}$ is the threshold power.

The raw data gathered by the sensor nodes will be modelated and as MFSK signal and send over the $2.4 \mathrm{GHz}$ channel. Due to the existance of foilage and other reflectors, the received signal will be faded and an outage probability will result depenmding on the signal power and the obstacles in the way of the trasmitted signal as the above equations modeled the trasmitted and received attenuated signal.

\section{MATERIALS AND METHODS}

Network nodes deployment: The spatial location of sensor node inside the greenhouse of $3 \times 6 \mathrm{~m}$ is shown in Fig. 4. The network was built with one sink node and four sensor nodes with three actuators nodes. The sink node hanged into the middle of greenhouse of $3.5 \mathrm{~m}$ above earth. The three actuators nodes were deployed 2 $\mathrm{m}$ height and connected directly to the actuators devices. The temperature-humidity sensor nodes deployed $1 \mathrm{~m}$ away of side wall and $2 \mathrm{~m}$ away from front-rear side of greenhouse. The fan actuator node function for ventilation is to activate the fan device. Using this spatial distribution provide covering of the all the area of the greenhouse. The other setup was built based on moving sink node inside the greenhouse with speed greater than 0 and less than Imper second.

Channel modeling: The mathematical model discussed above is used for performance prediction and evaluation of a communication system in generalized Rayleigh fading. Since the orientation and material properties of the obstacles between the transmitter and the receiver are not known in advance and may be time varying, hence, signals received can be characterized by stochastic random processes by Rayleigh/Ricain fading signal.

Sensors and network nodes: In this work, temperature and humidity sensor are deployed where their specification shown in Table 2, are employed. Sensor nodes chosen with fixed spatial location inside greenhouse. The main activity of the sensor nodes is to send periodically changes in temperature and humidity. The sink node of the network employs data recovery and saving of the network nodes data.

The experimental has been carried out based on the dense foliage, plants and movement of the sink node. The movement of the sink node is classified to be stationary or dynamic with speed less than Im/s. Stationary status environment implies there is only a dense foliage and plants that obstacle the radio propagation and cause signal losses and attenuation, while moving categorized as sink node mobility at speed greater than 0 and less than $0.5 \mathrm{~m} /$ sor footing the sink node with speed greater $0.5 \mathrm{~m} /$ sand less than $\mathrm{Im} / \mathrm{s}$. The dense trees and foliage affects the received signal widely, due to receiving of multiple versions of scattered components.

The multipath effect has been simulated and studied with LOS, NLOS, dense foliage and trees and with stationary or movement situations. The field of study carried out in an example of greenhouse of $18 \mathrm{~m}^{2}$ area. Wireless sensor network of star topology is built with sensor nodes of temperature-humidity sensor. The environment inside the greenhouse could be static or can be dynamic due to ventilation process by fans system.

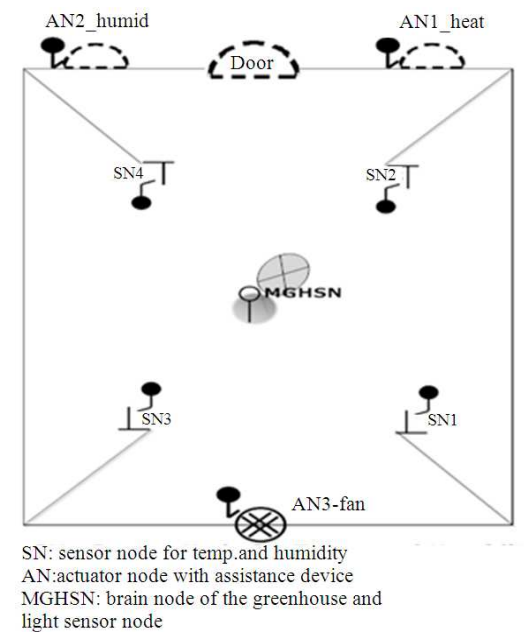

Fig. 4: Greenhouse network nodes spatial distribution 
Am. J. Applied Sci., 9 (1): 141-151, 2012

Table 2: Specification of SHT75 Temperature/humidity sensor

\begin{tabular}{lll}
\hline Parameter & Temperature sensor & Humidity sensor \\
\hline Resolution & $12 \mathrm{bit} / 0.04^{\circ} \mathrm{C}$ & 12 bit / $0.05 \% \mathrm{RH}$ \\
Accuracy & $\pm 0.3^{\circ} \mathrm{C}$ & $\pm 1.8 \% \mathrm{RH}$ \\
Operating Range & $-40^{\circ} \mathrm{C}-123^{\circ} \mathrm{C}$ & $0-100 \% \mathrm{RH}$ \\
Response Time & $5-30 \mathrm{sec}$ & $5-30 \mathrm{sec}$ \\
Source Voltage & $2.4 \mathrm{~V}-3.3 \mathrm{~V}$ & $2.4 \mathrm{~V}-3.3 \mathrm{~V}$ \\
Power & Sleep $2 \mu \mathrm{W}$, Measure $3 \mathrm{~mW}$ & Sleep $2 \mu \mathrm{W}$, Measure $3 \mathrm{~mW}$ \\
\hline
\end{tabular}

Table 3: A summary of the physical parameters of the simulation setup

\begin{tabular}{lll}
\hline Key Channel Parameters & Symbol & Typical value \\
\hline Carrier Frequency & $\mathrm{f}_{\mathrm{c}}$ & $900 \mathrm{MHz}$ \\
Digital Modulation & $\mathrm{f}_{\mathrm{m}}$ & MFSK \\
Number of tones & $\mathrm{M}$ & 8 \\
Number of bits & $\mathrm{N}$ & $3 \mathrm{bits} / \mathrm{symbol}$ \\
Number of symbols & $\mathrm{N}_{\mathrm{s}}$ & $50 \mathrm{symbol} / \mathrm{sec}$ \\
Frequency separation & $\Delta \mathrm{f}$ & $1 / \mathrm{T}_{\mathrm{s}}$ \\
Symbol time & $\mathrm{T}_{\mathrm{s}}$ & $0.02 \mathrm{sec}$ \\
Movement speed & $\mathrm{S}$ & $0-1 \mathrm{~m} \mathrm{sec}^{-1}$
\end{tabular}

The statistics of the received signal affected by multipath environment and movement was simulated. The carrier frequency used is $2.4 \mathrm{GHz}$, the modulation technique is the MFSK with eight tones that is three bits per symbol $\mathrm{N}=\log _{2}$ Mwhere $\mathrm{N}$ is number of bits per symbol.

The data rate chosen to be 150 bit per second, sourced by sensor with network node. In this study initially 50 symbols are taken, for orthogonal and non coherence receiver, with I/Tfrequency. Then the tone of the MFSK will be $\pm 25 \pm 75 \pm 125 \pm 175$ sing ( $2 \mathrm{i}-\mathrm{I}-\mathrm{M}) \Delta \mathrm{f} / 2$ The numbers of path and speed have been in two different scenarios. The simulation parameters are summarized in Table 3.

Stationary sink node status, the multipath varies between 2 and 40 . For each path, simulation was carried out for a time interval corresponding to 1250 wavelength. The received signal was generated using (6). The path amplitudes have been taken to be weibull distributed. The two parameter weibull distribution allowed the flexibility of making it easy to see the effects of varying scattering amplitudes. The phases $\phi_{\mathrm{i}}$, have been generated as uniform distributed random number between and $2 \pi$.

\section{RESULTS}

Figure 5 shows fading wave as per scenariol where the environment is static inside the greenhouse, the received signal fluctuated randomly with time with different rates of change and with less fading duration and cross level than when the dynamic status of environment.

Mobile sink node moves with speed range of 0-1 m $\mathrm{sec}^{-1}$. the simulation is carried out with same parameters setting of scenario one, with the including of Doppler shift $\pm f_{D}$ which is induce due to motion and its value is directly proportional to the velocity and direction of arrival of the received waves as shown in Figure 6. Simulation has been carried out by using (8).

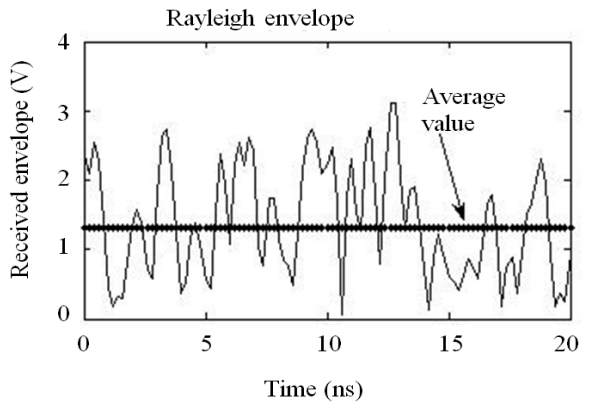

(a)

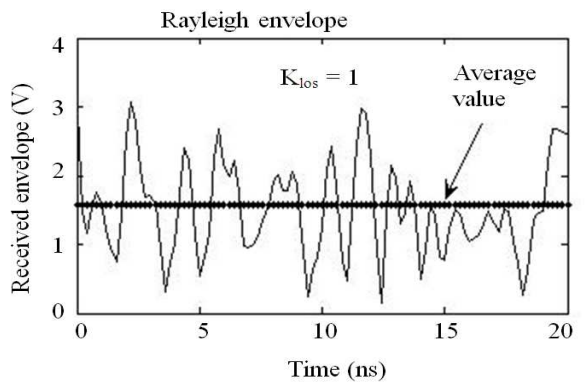

(b)

Fig. 5: The simulated rayleigh and Rician Faded RF signals and their envelopes
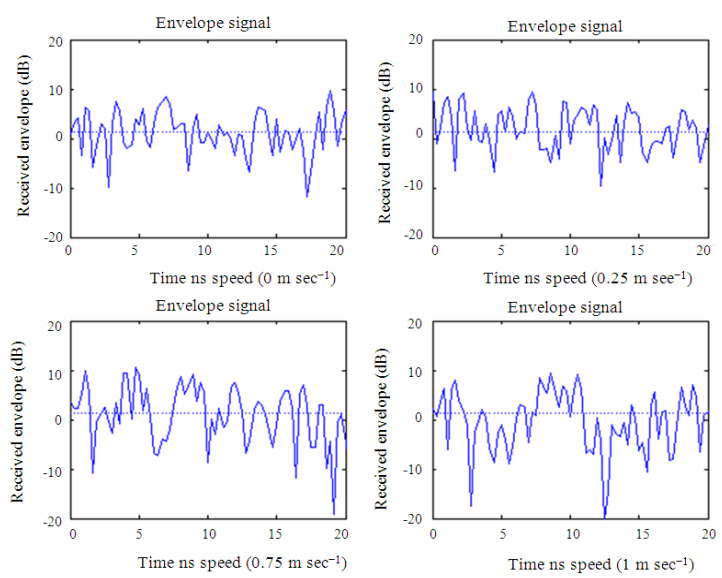

Fig. 6: The received faded signal due to doppler shift affects

Using programming method, the outage probability for a signal crossing a threshold level can be calculated by counting the number of times the received signal power goes below the threshold value then taking the ratio of the count divided by total number of samples.

Figure 7 shows outage probability curves for a threshold level of $-10 \mathrm{~dB}$. The outage probability calculated analytically and through simulations was found to be coinciding in their characteristics. 
Am. J. Applied Sci., 9 (1): 141-151, 2012
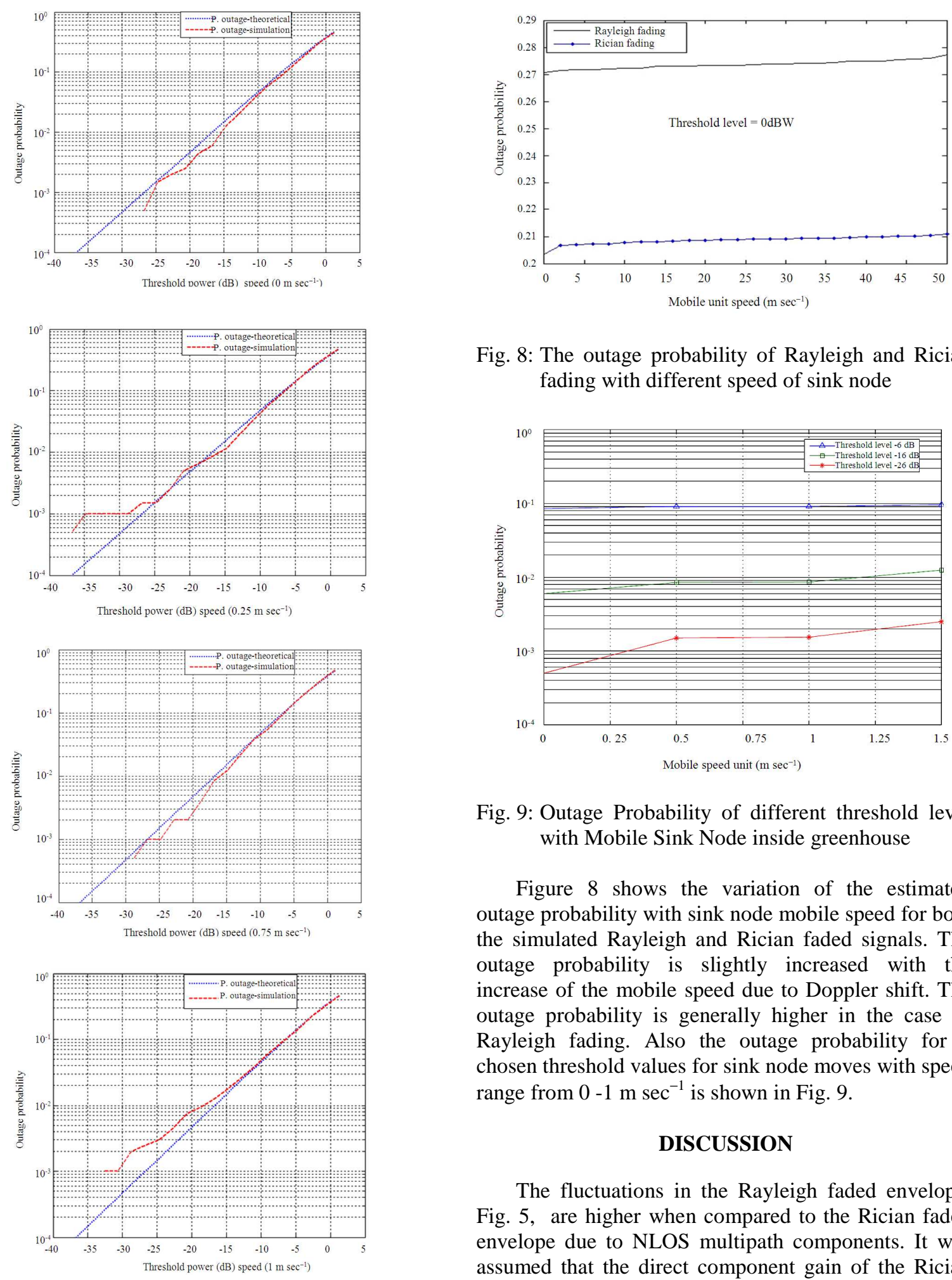

Fig. 8: The outage probability of Rayleigh and Rician fading with different speed of sink node

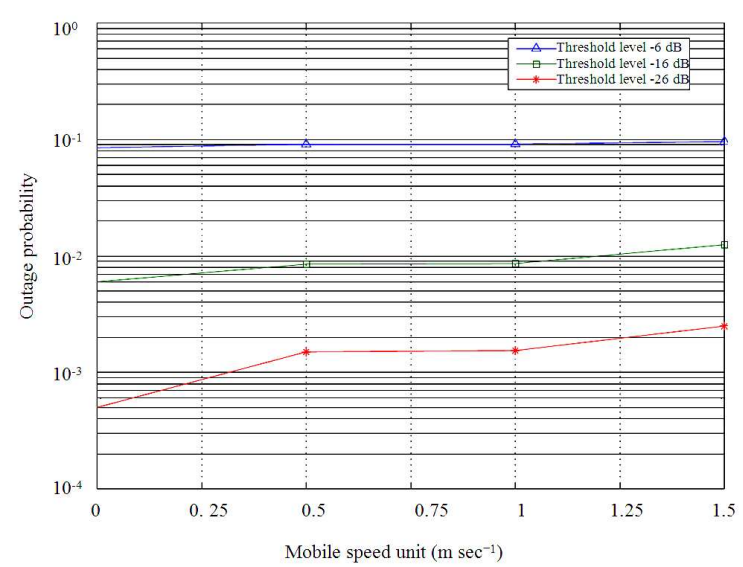

Fig. 9: Outage Probability of different threshold level with Mobile Sink Node inside greenhouse

Figure 8 shows the variation of the estimated outage probability with sink node mobile speed for both the simulated Rayleigh and Rician faded signals. The outage probability is slightly increased with the increase of the mobile speed due to Doppler shift. The outage probability is generally higher in the case of Rayleigh fading. Also the outage probability for 3 chosen threshold values for sink node moves with speed range from $0-1 \mathrm{~m} \mathrm{sec}^{-1}$ is shown in Fig. 9.

\section{DISCUSSION}

The fluctuations in the Rayleigh faded envelope, Fig. 5, are higher when compared to the Rician faded envelope due to NLOS multipath components. It was assumed that the direct component gain of the Rician channel $\operatorname{KLOS}=1$. Also the average value of the Rician received signal envelope is relatively higher than that of the Rayleigh envelope. Figure 6 shows three 
faded signals due to different speed of motion. When the mobile unit in scenario one, the average level crossing and the duration of fading is less than when it moving and the average received power is higher. When sink node is in stationary, the only affects of multipath fading is taken place. As the moving start the mobile unit will fused with frequency shift corresponding to the speed of movement, it's clear that within increased movement speed the received signal suffers from deep fading and highly fluctuation. As the nodes moves, the outage probability increased propotionaly with the speed of mobility. When the spatial location the mobile node could result in LOS status, the outage probability could be less due to the existance of LOS component, Fig. 7. Slightly increases of outage probability with increases of mobility of the sink node as shown in Fig. 8-9. As a result the sink node mobility did not affects clearly the performance of the network, this is due to low speed of the sink node. While the only main affects is the dense of the plants and foliage inside the greenhouse which cause of multipath fading of the received signal. When compare the performance of moving and stationary status of sink node inside the greenhouse with dense of foliage, it is found that mobility status with limit speed (less than 1 $\mathrm{m} \mathrm{sec}{ }^{-1}$ ) has better performance. This is due to the component of LOS that exist while moving contrary to fixed location that may cover and obstacles by dense of foliage.

The degrading effects of the Rician channel on the received signals are relatively smaller than those in the Rayleigh channel. The analysis shows that when speed of the mobile unit increased it affects totally the received signal at the receiver side due to multipath and the frequency shift parameters. The examined parameters included average received power level, rate of received signal fluctuations, outage probability and Doppler shift.

\section{CONCLUSION}

In this study, an approach to demonstrate wireless sensor network radio channel modeling based on multipath and Doppler Shift is presented. The experiment uses the dense foliage and plants inside greenhouse with network sink node either stationary or moving with speed $0<$ speed $<1 \mathrm{~m} \mathrm{sec}^{-1}$. The affects of various movement speed and multipath has been presented. It has been observed from experimental results that the received signal suffers rapid fluctuations and goes into deep fading when the speed increases as in movement of the sink node.
When there is a multipath phenomenon due to foliage and plants, the received signal will attenuate more than the situation of existence of the LOS component. Proper design of WSN based greenhouse is crucial demand prior to implementation, hence, for mobile wireless sensor network those affects must be studied and analyzed well for reliable and robust communication system.

For WSN application in agriculture and greenhouses, it is essential to pick the suitable distribution of the network nodes and the height of nodes so LOS component will highly possible between sensor nodes and the sink node. Indeed power consumption must well maintain so packet loss due to less power level can be overcome and the first component of the multipath will has high power over sensitivity of the receiver. Best position found is center of the application field of the greenhouse for the sink node in stationary situation, while for movement situation the best height of the sink node is to be little above line level of the sensor nodes.

\section{REFERENCES}

Anastasi, G., O. Farruggia, G.L. Re and M. Ortolani, 2009. Monitoring high-quality wine production using wireless sensor networks. Proceedinggs of the 42nd Hawaii International Conference on Systems Science, Jan. 5-8, IEEE Xplore Press, Big Island, HI, USA., pp: 1-7. DOI: 10.1109/HICSS.2009.313

Andersen, J.B., T.S. Rappaport and S. Yoshida 1995. Propagation measurements and models for wireless communications channels. IEEE Commun. Mag., 33: 42-49. DOI: 10.1109/35.339880

Andrade-Sanchez, P., F.J. Pierce and T.V. Elliot, 2007. Performance assessment of wireless sensor networks in agricultural settings. Proceedings of the 2007 ASABE Annual International Meeting, Minneapolis, MN, USA.

ANSI/IEEE Std 802.11, 1999. Wireless LAN Medium Access Control (MAC) and Physical Layer (PHY) Specifications. The Institute of Electrical and Electronics Engineers Inc., NY, USA.

Balamurugan, P. and K. Duraiswamy, 2011. Consistent and proficient algorithm for data gathering in wireless sensor networks. J. Comput. Sci., 7: 14001406. DOI: $10.3844 /$ jcssp.2011.1400.1406

Beaulieu, N.C. and C. Cheng, 2001. An efficient procedure for Nakagami-m fading simulation. Proceedings of the IEEE Global Telecommunications Conference, Nov. 25-29, IEEE Xplore Press, San Antonio, TX, USA., pp: 3336-3342. DOI: 10.1109/GLOCOM.2001.966304 
Bhattacharya, A., 2005. Digital Communication. 1st Edn., Tata McGraw-Hill Education, New Delhi, ISBN: 9780070591172, pp: 516.

Callaway, E.H., 2004. Wireless Sensor Networks: Architectures and Protocols. 1st Edn., Auerbach Publications, New York, USA., ISBN: 9780849318238, pp: 342.

Chomphan, S., 2010. Performance evaluation of multipulse based code excited linear predictive speech coder with bitrate scalable tool over additive white gaussian noise and rayleigh fading channels. J. Comput. Sci., 6: 1438-1442. DOI: 10.3844/jcssp.2010.1438.1442

Dargie, W. and C. Poellabauer, 2010. Fundamentals of Wireless Sensor Networks: Theory and Practice. 1st Edn., John Wiley and Sons Ltd., Chichester, West Sussex, U.K., Hoboken, NJ., ISBN: 0470997656, pp: 330.

Dersch, U. and R.J. Ruegg, 1993. Simulations of the time and frequency selective outdoor mobile radio channel. IEEE Trans. Vehicular Technol., 42: 338344. DOI: $10.1109 / 25.231886$

Haykin, S. and M. Moher, 2009. Communication Systems. 5th Edn., John Wiley Sons, Hoboken, N.J., ISBN: 0470169966, pp: 422.

Hebel, M.A., 2006. Meeting wide-area agricultural data acquisition and control challenges through ZigBee wireless network technology. Proceeding of the 4th World Congress Conference on Computers in Agriculture and Natural Resources, Jul. 24-26, Orlando, Florida USA.

Hebel, M.A., R.F. Tate and D.G. Watson, 2007. Results of wireless sensor network transceiver testing for agricultural applications. Proceedings of the 2007 ASAE Annual International Meeting, Minneapolis, MN, USA.

Ismail, N.M.H. and M. Othman, 2009. Low power phase locked loop frequency synthesizer for 2.4 $\mathrm{GHz}$ band zigbee. Am. J. Eng. Applied Sci., 2: 337-343. DOI: 10.3844/ajeassp.2009.337.343

Kirubanand, V.B. and S. Palaniammal, 2011. Study of performance analysis in wired and wireless network. Am. J. Applied Sci., 8: 826-832. DOI: 10.3844/ajassp.2011.826.832

Mainwaring, A., D. Culler, J. Polastre, R. Szewczyk and J. Anderson 2002. wireless sensor networks for habitat monitoring. Proceedings of the 1st ACM International Workshop on Wireless Sensor Networks and Applications, Sept. 28-28, Atlanta, GA, USA., pp: 88-97. DOI: $10.1145 / 570738.570751$
Melodia, T., D. Pompili, V.C. Gungor and I.F. Akyildiz, 2007. Communication and coordination in wireless sensor and actor networks. IEEE Trans. Mobile Comput., 6: 1116-1129. DOI: 10.1109/TMC.2007.1009

Papoulis, A., 1991. Probability, Random Variables and Stochastic Processes. 3rd Edn., McGraw Hill, New York, ISBN: 007066465X, pp: 666.

Patel, R.B., V.K. Katiyar and V. Garg, 2006. Mobile agents in wireless LAN and cellular data networks. J. Comput. Sci., 2: 410-418. DOI: 10.3844/jcssp.2006.410.418

Sabri, N., S.A. Aljunid, R.B. Ahmad, A. Yahya and R. Kamaruddin et al., 2011. Wireless sensor actor network based on fuzzy inference system for greenhouse climate control. J. Applied Sci., 11: 3104-3116.

Shan, Q., Y. Liu, G. Prosser and D. Brown, 2004. Wireless intelligent sensor networks for refrigerated vehicle. Proceedings of the IEEE 6th Symposium on Emerging Technologies Mobile and Wireless Communication, May 31-Jun. 2, IEEE Xplore Press, China, pp: 525-528. DOI: 10.1109/CASSET.2004.1321941

Sklar, B., 1997. Rayleigh fading channels in mobile digital communication systems-Part I: Characterization. IEEE Commun. Mag., 35: 90100. DOI: $10.1109 / 35.601747$

Sohraby, K., D. Minoli and T.F. Znati, 2007. Wireless Sensor Networks: Technology, Protocols and Applications. 1st Edn., John Wiley and Sons, Hoboken, NJ., ISBN 0471743003, pp: 307.

Tate, R.F., M.A. Hebel and D.G. Watson, 2008. WSN link budget analysis for precision agriculture. Providence, Rhode Island.

Tse, D. and P. Viswanath, 2005. Fundamentals of Wireless Communication. 1st Edn., Cambridge University Press, Cambridge, ISBN 0521845270, pp: 564.

Willig, A., K. Matheus and A. Wolisz, 2005. Wireless technology in industrial networks. Proc. IEEE, 93: 1130-1151. DOI: 10.1109/JPROC.2005.849717

Zheng, Y.R. and C. Xiao, 2003. Simulation models with correct statistical properties for rayleigh fading channels. IEEE Trans. Commun., 51: 920-928. 